\title{
Voyageurs et polygraphes autrichiens autour de 1809
}

\section{Françoise Knopper}

\section{(2) OpenEdition}

\section{Journals}

\section{Édition électronique}

URL : http://journals.openedition.org/recherchestravaux/471

DOI : 10.4000/recherchestravaux.471

ISSN : 1969-6434

\section{Éditeur}

UGA Éditions/Université Grenoble Alpes

\section{Édition imprimée}

Date de publication : 15 décembre 2011

Pagination : 17-33

ISBN : 978-2-84310-215-8

ISSN : 0151-1874

\section{Référence électronique}

Françoise Knopper, «Voyageurs et polygraphes autrichiens autour de 1809 », Recherches \& Travaux [En ligne], 79 | 2011, mis en ligne le 15 juin 2013, consulté le 07 septembre 2020. URL : http:// journals.openedition.org/recherchestravaux/471; DOI : https://doi.org/10.4000/recherchestravaux 471 
Françoise KnOPPER

Université Toulouse - Le Mirail

\section{Voyageurs et polygraphes autrichiens autour de 1809}

Les années 1806 à I8Io correspondent moins à un léger ralentissement dans la parution de relations de voyage allemandes, qui restent fort nombreuses ${ }^{\mathrm{I}}$, quà une période d'hésitation voire de transition en ce qui concerne les auteurs germanophones relevant de la catégorie des chroniqueurs professionnels, des polygraphes dits "Reiseschriftsteller». Ce constat vaut aussi bien pour ceux d'entre eux qui résidaient en Autriche et décrivaient leur patrie que pour les visiteurs qui écrivaient sur l'Autriche.

Les années fastes, celles où les relations de voyage avaient inondé le marché, avaient correspondu à trois grandes vagues, étudiées par Peter J. Brenner, Wolfgang Griep, Hans-Wolf Jäger ${ }^{2}$ ou encore par Michael Maurer dans son bilan interdisciplinaire 3 . Ces chercheurs ont appréhendé le récit de voyage en tant que genre à part entière, avec ses particularités, ses limites, son évolution dans les années 1780 , et sa réception immédiate.

I. La multitude des titres parus à cette époque est déjà consignée dans les anciennes bibliographies comme celles de W. Heinsius, E. Cox, W. Engelmann, W. Griep, P. E. Richter, J. von Sartori, A. Schlossar, G. H. Stuck.

2. P. J. Brenner, Der Reisebericht in der deutschen Literatur. Ein Forschungsüberblick als Vorstudie zu einer Gattungsgeschichte, Tübingen, Niemeyer, I990.

3. M. Maurer (éd.), Neue Impulse der Reiseforschung, Berlin, Akademie Verlag, 1999. Sur les apports récents de l'histoire sociale, de l'imagologie, des transferts culturels et de l'interdisciplinarité en relation avec la littérature autrichienne des voyages, voir aussi Fr. Knopper, "Présences autrichiennes dans la littérature des voyages jusqu’à la fin du Xix ${ }^{e}$ siècle», Littérature de voyage - Regards autrichiens sur le monde, études réunies par J. Benay et J. Lajarrige, Austriaca, no 62, 2007, p. 49-66. 
Durant la première vague, les représentants des Lumières tardives ${ }^{4}$, qui s'étaient tenus sur la défensive, venaient jusqu'à Vienne pour resserrer leurs rangs (I780 à I784). Leur chef de file, le libraire berlinois Friedrich Nicolai, avait publié une longue description de son voyage en Allemagne du Sud et en Autriche où il dénonçait de façon obsessionnelle un regain du prosélytisme catholique qui risquait, selon lui, de fragiliser la Prusse et le rationalisme de ses luthériens néologues. La vague suivante se nourrit des enquêtes que les "pèlerins de la liberté» menèrent dans le Paris révolutionnaire (I789 à 1792). Enfin, le genre des relations connut une recrudescence dans les années I80I à 1803 , une fois la paix signée, la rive gauche du Rhin annexée à la France et les remaniements territoriaux du Saint-Empire irréversiblement engagés ${ }^{5}$. Il n'en demeure pas moins que l'engouement des lecteurs et des lectrices pour les relations de voyage perdura ${ }^{6}:$ une vingtaine de titres correspondant à la description de l'Autriche ont été répertoriés à ce moment-là dans la bibliographie du Journal général de la littérature étrangère ${ }^{7}$.

Mais il se manifestait depuis le tournant du siècle un certain flottement à l'intérieur du genre. Ce dernier avait jusqu'alors été codifié en fonction de rubriques obligatoires (climat, démographie, nature des institutions, ressources et productions, vie culturelle et caractère des habitants). Les récits de voyage des Aufklärer avaient une dimension informative voire encyclopédique, leur vocation était de répercuter les revendications des intellectuels locaux, de marquer leur solidarité, de tisser ou resserrer des réseaux de sociabilité. Or ces critères ne répondaient plus aux attentes de la nouvelle génération qui adhérait dorénavant à l'idéalisme des classiques de Weimar ou à celui des premiers romantiques et ne se contentait plus d'une approche empirique. Une nouvelle école, celle des chroniques esthétisantes, se fondait sur l'interaction du sujet qui écrit et de l'objet qui est décrit : pour l'orientation classique, la référence est le Voyage en Italie de Goethe, pour qui le spectacle immédiat de l'environnement procure une maturité et un bien-être durables; pour l'orientation romantique, ce sont Wackenroder et Tieck qui

4. R.-R. Wuthenow, Die erfahrene Welt, europäische Reiseliteratur im Zeitalter der Aufklärung, Francfort-sur-le-Main, Insel, 1980; W. Stewart, Die Reisebeschreibung und ihre Theorie im Deutschland des I8. Jahrhunderts, Bonn, Bouvier, I978.

5. Fr. Knopper, A. Ruiz (éd.), Les Voyageurs européens sur les chemins de la guerre et de la paix du temps des Lumières au début du XIX siècle, Bordeaux, Presses universitaires de Bordeaux, 2006.

6. H. Mayrhofer (Die Reise in der Prosaliteratur in Österreich von I800-I850 : ein Beitrag zur Erforschung der literarischen Zweckformen, Phil. Diss. Vienne, 1978) recense une centaine de relations écrites par des auteurs autrichiens entre i8I5 et I848.

7. Journal général de la littérature étrangère ou Indicateur bibliographique et raisonné des livres nouveaux en tous genres, Paris-Strasbourg, Treuttel, I805, 5 année, $\mathrm{I}^{\mathrm{re}}$ partie, p. 562-563. 
ont problématisé la notion de perception synthétisante dès I793, lors de leurs périples en Franconie.

La mixité s'imposait cependant dans la majorité des relations écrites au début du XIx ${ }^{e}$ siècle. Le contenu des observations transmises restait en effet topographique, économique, sociologique, mais les grilles de perception, elles, avaient été réajustées et impliquaient la prise en compte de la vie culturelle populaire, la prédilection pour le sublime, l'exaltation de la sensibilité religieuse et l'admiration de paysages escarpés. En définitive, il faudra attendre les années $1820^{8}$, avec les Tableaux de voyage d'un Willibald Alexis ou ceux d'un Heinrich Heine, pour basculer dans un autre type d'écriture et faire triompher l'émancipation du sujet.

L'actualité elle-même pouvait aussi conforter les auteurs dans une certaine incertitude, l'imminence d'une reprise de la guerre contre Napoléon étant perceptible en Autriche9. Durant toute la première décennie du XIX ${ }^{\mathrm{e}}$ siècle, les voyageurs qui se rendaient à Vienne ou se promenaient en Autriche pouvaient observer qu'aussi bien en I805 qu'en I809, l'entourage de l'empereur François I ${ }^{\mathrm{er}}$, en particulier son frère l'archiduc Charles, souhaitait recourir à des solutions militaires et susciter un sursaut général, ce qui les frappait d'autant plus que cela ne leur semblait pas être le cas dans d'autres régions d'Allemagne.

C'est pourquoi, quand le chercheur applique ces considérations générales à l'Autriche de cette époque, il est confronté à une série de questions. La première porte sur la manière de délimiter l'aire culturelle "autrichienne» : s'agit-il de l'ensemble des territoires administrés par les Habsbourg ou seulement des parties germanophones? est-il judicieux d'y associer Prague et la Bohême qui faisaient partie de l'ancien Saint-Empire? Le plus pertinent nous a paru ici de nous en tenir à des régions qui étaient considérées sans conteste comme représentatives de l'empire d'Autriche, à savoir Vienne et les Alpes autrichiennes. Et pour l'appartenance culturelle des auteurs, nous nous en tiendrons à leurs propres assertions sur leur identité. La deuxième question porte sur les catégories professionnelles les plus répandues chez les voyageurs ayant alors décrit ces régions d'Autriche : les étrangers étaient surtout des militaires, des diplomates, des artistes; les auteurs autrichiens qui ont tenu à exprimer leur patriotisme en décrivant leur pays au tournant du siècle

8. Voir entre autres E. Jost, Landschaftsblick und Landschaftsbild. Wahrnehmung und Ästhetik im Reisebericht 1780-I820, Freiburg, Rombach, 2005; Chr. Ujma (éd.), Wege in die Moderne. Reiseliteratur von Schriftstellerinnen und Schriftstellern des Vormärz, Bielefeld, Aisthesis, 2009.

9. Sur les mémoires écrits par des officiers autrichiens en I809 et I8IO, voir J. Sachslehner, Napoleon in Wien. Fakten und Legenden, Vienne-Graz-Klagenfurt, Pichler, 2008. 
étaient majoritairement des scientifiques (surtout des minéralogistes et des botanistes $\left.^{\mathrm{r}}\right)$, des ecclésiastiques érudits, des enseignants, et - dans une veine très viennoise - des humoristes. Ce qui mérite enfin de retenir l'attention est la tradition du voyage (et de sa relation) parmi les archiducs d'Autriche; c'est pourquoi nous retiendrons l'exemple de l'archiduc Jean, contemporain de Stendhal.

Notre objectif sera, tout en respectant l'hétérogénéité intrinsèque à ce genre d'écriture, d'examiner quelle est la cohérence interne des notes que le chroniqueur a prises au quotidien et ensuite remaniées en vue d'une publication. Étant donné le bon accueil que le lectorat réservait à cette littérature de voyage, chacun s'efforçait d'être utile aux historiens, géographes ou musicologues, de collecter des informations que seuls les visiteurs avaient l'occasion d'observer sur place, lors de leurs conversations, et de les noter quotidiennement. L'interprétation est parfois malaisée car, pour repérer l'orientation propre à ces auteurs, pour cerner leur vision du monde et de la société, il est nécessaire de formuler des hypothèses sur le bagage culturel de chacun et sur l'implicite qui sous-tend leurs descriptions, lesquelles, indéniablement, sont utilitaires, énumératives. À cela s'ajoutent des critères factuels tels que le trajet suivi, la datation, ainsi que les facteurs narratologiques, car chaque auteur essayait de répondre à l'horizon d'attente de son lectorat et prétendait être un témoin fiable, un observateur polyvalent, un écrivain à la fois instructif et divertissant, un narrateur sachant parler simultanément des autres et de lui-même.

\section{Arndt et Reichardt}

Parmi les chroniqueurs venus d'Allemagne du Nord et qui ont décrit la vie à Vienne à la veille de l'arrivée de Napoléon, on peut mentionner les noms d'Arndt et de Reichardt dont les relations sont particulièrement typées et exhaustives. Leur brève présentation aidera à mettre ensuite en perspective les exemples autrichiens que nous allons privilégier dans cet article.

Ernst Moritz Arndt (I769-I860), poète qui associera le messianisme romantique à l'exaltation nationaliste et deviendra le propagandiste attitré de la Prusse en I8I3, lors des guerres d'indépendance, est encore étudiant à l'époque de son voyage. Il relate sa visite de Vienne en 1798 sur un mode irrévérencieux, affectionnant les paradoxes, se livrant à une opération généralisée de démystification, qualifiant par exemple l'empereur de "premier habitant

Io. Voir les indications bibliographiques données par M. de Serres dans son Voyage dans l'empire d'Autriche pendant les années I809 et I8I0, Arthus Bertrand, I8I4, en particulier p. XLI-LXIII. 
de Vienne ${ }^{\mathrm{II}}$ », en somme primus inter pares. Il ne dénigre cependant pas systématiquement la ville, et sa description se veut avant tout variée, vivante, contrastée. Avant de regrouper ces remarques de façon thématique, il visite un quartier après l'autre, fait promener le lecteur dans les artères principales, le long des monuments célèbres, s'attarde sur le mélange de tous les milieux qu'il trouve plaisant, par exemple au théâtre Marinelli. Il prétend visiter la cathédrale pour dissiper les vapeurs du vin hongrois dont il a abusé mais donne en fait plus de détails sur le bâtiment que bien d'autres avant lui. Les rubriques qui suivent concernent la sociabilité : cafés, fiacres, surveillance policière, université. Les arts occupent une grande place : Arndt décrit les richesses du musée impérial abrité par le Belvédère, réserve un chapitre à la musique, en particulier à Mozart, un autre aux différents théâtres et aux acteurs, aux opéras et ballets. Il présente ensuite, en les classant de façon provocatrice, diverses catégories sociales : prostituées, militaires, êtres humains, cour et courtisans, puis paysans.

Ce texte se singularise par la vitalité du narrateur et la fermeté de son écriture. La structure de l'ensemble s'apparente à celle d'une œuvre d'art totale qui mélange les genres. Les jugements portés sont ceux d'un cosmopolite effronté, curieux, non conformiste mais intéressé par toutes les formes de cultures. Dans l'histoire du genre, il est une variante romantique de la tradition des récits jadis écrits par les aventuriers du XviII ${ }^{\mathrm{e}}$ siècle tels Merveilleux ou Pöllnitz : les anecdotes savoureuses, parfois picaresques, côtoient des informations précises et des descriptions réalistes.

Les Lettres dans lesquelles le chef d'orchestre et compositeur Johann Friedrich Reichardt (I752-18I4) relate son séjour à Vienne à la fin de l'année I808 et au début de 1809 sont pour leur part d'une remarquable richesse informative sur la vie artistique du moment, sur les créations musicales et théâtrales les plus récentes, et - ce qui est particulièrement précieux - sur le jeu des acteurs. Reichardt garde une vive admiration pour le vieux Haydn auquel il rend visite, tout comme à Beethoven qui lui paraît très isolé, et, comme on peut s'y attendre, il commente la virtuosité requise par les opéras de Mozart, notamment dans Don Juan. Ses Lettres entrent dans la catégorie - redécouverte il y a peu ${ }^{12}$ - des voyageurs dits "musicaux» dont l'un des éminents représentants avait été l'Anglais Charles Burney en 1772. Concession aux usages de l'époque, ces prétendues Lettres sont en fait un journal intime selon la formule que Reichardt avait déjà pratiquée avec bonheur en 1792 à l'occasion de son

II. E. M. Arndt, Reisen durch einen Theil Deutschlands, Ungarns, Italiens und Frankreichs, Erster Theil, Leipzig, Gräss, I804, p. I70.

I2. H. E. Bödeker, P. Veit, M. Werner, Le concert et son public : mutations de la vie musicale en Europe de I780 à I9I4 (France, Allemagne, Angleterre), Éditions de la Maison des sciences de l'homme, 2002. 
séjour à Paris. À Vienne, où Reichardt se dit traité en éminent critique d'art et reçu dans les salons des plus hauts personnages de l'État, il fait connaître au grand public le nom des mécènes viennois et, lui qui avait jadis été un adepte de la Révolution, vante tous leurs talents. Il ne côtoie pas uniquement ce public distingué, il signale aussi quels sont les autres voyageurs étrangers, généralement nobles, qu'il rencontre, ce qui permettrait au chercheur, s'il consultait les notes de voyage de ces derniers, de mener une étude intertextuelle inédite.

Ces deux exemples, si différents soient-ils, correspondent à une évolution de l'écriture du voyage en Allemagne au tournant du siècle : le sujet individuel ne s'efface plus derrière les informations qu'il donne, alors que, dans les générations précédentes, les chroniqueurs tendaient à faire passer leurs témoignages pour une photographie de la réalité observée. Bien au contraire, Arndt et Reichardt ramènent tout à leur personne et à leurs impressions; leur état des lieux est autocentrique.

Par rapport à ces deux ouvrages de grande qualité, les exemples proposés ci-dessous sembleront plus modestes. Nous les avons choisis d'une part en fonction de la résidence et de l'origine géographique des auteurs, qui sont des Autrichiens de naissance ou d'adoption, afin de donner un aperçu de quelques personnalités que Stendhal était susceptible de lire ou de croiser à Vienne. D'autre part, les textes sélectionnés ont connu une large diffusion et ont servi de guides. De plus, leur enjeu était symptomatique des préoccupations professionnelles et du besoin de réformes souhaités par ces auteurs.

\section{Topographies écrites par des autochtones}

Les topographies étaient des textes de référence destinés aux voyageurs. Elles pouvaient se lire à la manière des reportages culturels des magazines d'aujourd'hui ou être consultées à titre de guides. Une particularité de ce genre de textes, qui se retrouve d'ailleurs dans l'ensemble de l'Allemagne ${ }^{13}$, était de riposter aux critiques : la description des lieux par les autochtones résultait souvent de l'intention de répliquer à des visiteurs mécontents ou à des autochtones qui avaient publié des propos railleurs sur Vienne ou sur leurs provinces respectives ${ }^{\mathrm{I}}$.

I3. Fr. Knopper, "Öffentlichkeit und Meinungsfreiheit. Repliken und Gegenschriften zu Reisebeschreibungen am Ausgang des 18. Jahrhunderts", dans Die Welt erfahren. Reisen als kulturelle Begegnung von I780 bis heute, A. Bauerkämper, H. E. Bödeker, B. Struck (éd.), Francfort-New York, Campus, 2004, p. 219-238.

I4. Les échanges de ripostes existaient aussi entre provinces : il y eut en I8II à propos de la Carinthie un vif débat entre le médecin styrien Sartori, auteur d'une relation de voyage très critique, et Enzensberg, haut fonctionnaire à Klagenfurt. 
C'est le cas de Johann Pezzl (I756-I823), écrivain et plus tard bibliothécaire, auteur d'un Voyage dans le cercle de Bavière (I784). Ses positions anticléricales virulentes et sa sympathie pour l'Ordre des Illuminés avaient valu à Pezzl de devoir s'exiler de sa Bavière natale et de se réfugier à Zürich puis à Salzbourg, et finalement à Vienne. Son Esquisse de Vienne fut publiée une première fois en I787-I790, rééditée une quatrième fois en I803 légèrement remaniée. Cet ouvrage qui a eu une exceptionnelle longévité a été maintes fois réédité au XIX ${ }^{e}$ siècle. Divisé en six brochures plus tard regroupées en un volume, inspiré des Tableaux de Paris de Mercier, très souvent satirique, ce texte est représentatif de l'Aufklärung catholique. Les remaniements apportés en 1803 sont le signe de la prudence post-révolutionnaire et plus précisément de la distance prise par rapport aux quelques jacobins autrichiens. Pezzl gomme en 1803 les passages les plus irrévérencieux envers l'Église et toutes les attaques qui pourraient être ressenties comme une critique du système monarchique. Ces suppressions mises à part, la topographie de Pezzl reste tout à fait placée sous le signe des Lumières tardives et centrée sur le milieu urbain. En dépit de l'emploi malicieux de stéréotypes indulgents, parfois critiques et pédagogiques, notamment concernant la «folie» du jeu ou les querelles entre quartiers, le ton est globalement élogieux, par exemple dans les pages où Pezzl expose que chaque "classe» de l'humanité, qu'il s'agisse des responsables politiques, ou militaires, d'artistes, de commerçants, de fonctionnaires ou d' "individualistes", pourra trouver à Vienne le milieu qui lui correspond et le soutien officiel dont elle aurait besoin ${ }^{15}$.

Que ce soit dans les chapitres démographiques, géographiques, économiques, ou dans les pages culturelles, attendues dans une topographie, Pezzl relaie systématiquement la propagande joséphiste dont il avait été un des premiers adeptes. Par exemple, quand il dénombre les diverses nationalités installées à Vienne, détaille leurs activités et leurs implantations, ce n'est pas seulement en démographe qu'il travaille, mais c'est pour vanter la tolérance religieuse du gouvernement et signaler par quels canaux les écrits les plus novateurs des médecins et des philosophes pouvaient circuler hors d'Europe.

Outre cette propagande joséphiste, Pezzl ne perd pas une occasion pour réagir aux critiques des visiteurs mécontents qui avaient publié à leur retour des propos railleurs sur Vienne. Il ne se prive pas, en I803, d'ajouter quelques piques contre le spiritisme à la mode dans le Berlin de Frédéric-Guillaume II. Et aux esprits chagrins qui attribuaient la légendaire hospitalité viennoise

I5. J. Pezzl, Skizze von Wien, unter der Regierung Joseph des Zweyten, Vienne, J. D. Degen, I803, p. I7-I9. 
à un déplorable penchant au luxe et au gaspillage, il rétorque - et il n'est pas le seul - qu'il s'agit d'une «vertu patriarcale» et d'un «irrépressible élan du cœur", s'abritant derrière les témoignages, à l'époque très connus, des touristes anglais Sherlock, Moore, Coxe, ou des Allemands Archenholz, Wekhrlin et Nicolai. Il suggérait ainsi que la sociabilité était authentique, voire germanique, et n'avait rien de commun avec la frivolité parisienne ${ }^{16}$. En définitive, le plaidoyer pro domo de Pezzl fournit un intéressant témoignage de la réussite de son assimilation puisque l'Autriche était devenue sa patrie d'adoption. Bien que restant dans la tradition de la Spätaufklärung, cette assimilation se confirme dans les remaniements politiquement corrects qu'il a apportés à la réédition de sa topographie.

Une autre conception du tourisme se fait jour dans les Excursions et promenades autour de Vienne ${ }^{17}$ du pédagogue Franz Anton de Paula Gaheis (1763-1809), originaire de Krems. Elle illustre l'évolution des habitudes de la bonne société viennoise, que Gaheis incite à s'instruire sur le patrimoine local. L'optique n'est ni utilitaire ni polémique, elle reflète plutôt un souci de conserver le patrimoine par le biais d'une mise en perspective à la fois historique et esthétique.

Ce texte, fut loué par la presse allemande. Il propose une série d'excursions d'une à deux journées environ. Elles sont l'équivalent, peut-être le modèle, des Promenades dans la Marche de Brandebourg écrites par Fontane soixante-dix ans plus tard. Gaheis décrit avec minutie les endroits traversés, sans différencier le détail éphémère et ce qui pourrait être primordial, l'essentiel étant de faire converger connaissances de l'histoire locale et loisirs touristiques. Il veille à varier la présentation des lieux, faisant alterner ceux qui ont un intérêt urbanistique et ceux dont la vocation est agricole. Le narrateur s'arrête volontiers dans les cimetières pour contempler la pierre tombale d'un bourgeois apprécié par ses concitoyens et décédé depuis peu. Il donne plus loin de nombreuses informations sur l'agriculture, les fermes, les champs. Il se rend dans la moindre chapelle, reconstituant la date et les circonstances de sa fondation, et recopiant les inscriptions qu'on peut y lire. Il insiste sur les divertissements offerts, et une excursion est consacrée à la station thermale de Baden. Dans l'ensemble, cet ouvrage tend à démontrer que la limite entre

16. Ibid., p. 47. Sur la "bourgeoisie» viennoise regroupant gens d'affaires et intellectuels, voir I. Mittenzwei, Zwischen gestern und morgen : Wiens frühe Bourgeoisie an der Wende vom I8. zum I9. Jahrhundert, Vienne-Cologne-Weimar, Böhlau, I998.

17. Wanderungen und Spazierfahrten in die Gegenden um Wien, éd. Fr. de Paula Gaheis, t. I, Doll und Schuender, I798, rééd. en I808, Vienne, 72 brochures regroupées en 3 volumes, parfois pourvus d'index. 
la ville de Vienne et la campagne environnante est souvent à peine perceptible, de sorte que la nature paraît accueillante, habitée, parfois semblable à un théâtre où les promeneurs viennent deviser, comme à la Brigittenau : l'atmosphère paisible rappelle les estampes de l'époque avec les parcs des nobles demeures s'ouvrant sur de larges perspectives. Les châteaux visités, comme celui de Cobenzl, donnent à penser que les lecteurs concernés appartenaient à la bonne société, à une société cultivée, telle que la souhaitait Gaheis qui reste pédagogue : le classicisme le plus exigeant sert d'arrière-plan à ces délassements dominicaux car le narrateur rapporte que lui et ses compagnons discutent, tout en se promenant le long du Danube, des Horen et des Xenien, donc de Goethe et Schiller.

Il échafaude en quelque sorte une éducation à la culture du loisir, qui incite les citadins à la sérénité sans perdre de vue la gravité du moment. Le narrateur, à l'occasion d'une fête populaire champêtre, décrit par exemple la variété des amusements proposés mais insiste sur la distance à garder :

Un peu plus bas, un homme avait une lanterne magique. Ceux qui étaient trop jeunes pour aller danser s'amusaient à regarder les images que cet homme qui leur semblait tout-puissant faisait défiler sous leurs yeux. Ce qu'il montrait le plus souvent, c'étaient les batailles et les sièges tenus durant la dernière guerre, et le fascinant Bonaparte. Et il s'entendait parfaitement à inciter à la réflexion ${ }^{18}$ !

Sans autre commentaire, le narrateur s'amuse ensuite à singer les vendeurs de salami italien et leur salmigondis. Un violoniste aveugle éveille sa pitié. Bref, des scènes de genre miniaturisées qui servent de prélude au Biedermeier.

La grande leçon que le lecteur est censé tirer est contre-révolutionnaire : il importerait de rendre hommage à la noblesse, très influente à la cour et protectrice des arts, tout en témoignant de l'harmonieux mélange des milieux sociaux sur les bords du Danube :

Et ce qui augmentait l'éclat de ce spectacle, était le fait que vers le coucher du soleil il y eut aussi des personnes de milieux cultivés qui vinrent se mêler en toute simplicité à la joyeuse populace; l'expression plus intellectuelle de leur visage, la distinction naturelle de leur attitude, leur participation spontanée et distinguée aux différents spectacles, leur conversation choisie, conféraient à l'ensemble de la fête une dignité non guindée, bien éloignée de la grandezza espagnole, un raffinement et un esprit plus cultivé, sans même le faire délibérément. Les blagues douteuses, que le gros rire de l'homme du peuple qui en est l'auteur finit par rendre toutefois intéressantes, étaient compensées par les commentaires pertinents de ces personnes éminentes ${ }^{19}$

18. Ibid., Wanderungen, t. I, $6^{\mathrm{e}}$ promenade, Brigittenau, p. 27.

19. Ibid., p. 36. 


\section{Excursions patriotiques}

Ce conservatisme souriant était complété par des relations de voyage plus techniques qui relèvent d'un autre type de documents, les excursions patriotiques, qui avaient été initialement conçues sur le modèle des homes travels anglais du $\mathrm{XVIII}^{\mathrm{e}}$ siècle $^{20}$. Ces récits restent un des genres les plus répandus en Autriche après I80o. Ils sont souvent rédigés par des botanistes ou des minéralogistes dont les disciplines ne cessaient de se développer. Deux ouvrages sont volontiers cités par les contemporains, celui de Schultes et celui de Unger. Dans ses Excursions, Josef August Schultes (1773-I83I) enquête, à pied, sur la topographie, la population, les richesses naturelles, agricoles, minières, manufacturières d'une montagne de Basse-Autriche, proche de Vienne, le Schneeberg ${ }^{21}$. Ce naturaliste viennois, qui est aussi médecin, s'ingénie à fournir les informations susceptibles de servir de publicité et de caution aux fonctionnaires et réformateurs des endroits visités, et ses critères rester ceux des géographes et des voyageurs caméralistes des années 1780 . Schultes introduit cependant aussi une autre composante, qui sera dorénavant présente dans toutes les relations des années I800 : la conviction que le voyageur devrait définir des types nationaux, des traits caractéristiques, et ce, dans le cas de l'Autriche, afin de répondre aux exigences des patriotes attachés à leur système monarchique. Il reste au demeurant pragmatique et, comme d'autres médecins allemands qui visitent ces régions à la même époque ${ }^{22}$, il n’idéalise pas systématiquement les milieux campagnards, distingue entre les habitants des plaines et les montagnards, formule des observations médicales, interprète scientifiquement le prétendu crétinisme des Alpes, dénonce les déficits d'éducation scolaire.

Conformément au goût du dialogue et de la réplique instauré entre voyageurs, un autre Autrichien, Johann Carl Unger, prend la route en I8O2 pour, entre autres, confirmer ou infirmer les observations de Schultes. Unger (I74II836), d'origine hongroise, était juriste et professeur à l'Académie militaire. Lui aussi s'inscrit encore dans la tradition de l'Aufklärung: il se réfere d'ailleurs à une Topographie parue en 1769 , celle de Weiskern. Ses déplacements sont placés sous le signe de la méticulosité, les noms des hameaux se succèdent, comme sur une carte. Ses notes se veulent savantes et actualisées, sa présentation des thermes de Baden fait le bilan des découvertes récentes de Volta et Geusau. Il s'insurge contre tout ce qui lui parait relever de l'imagination,

20. Fr. Knopper, Le Regard du voyageur en Allemagne et en Autriche 1775-1803, Nancy, Presses universitaires de Nancy, 1992, p. 84 et suiv.

2I. J. A. Schultes, Ausflüge nach dem Schneeberge in Unterösterreich, Vienne, Degen, I802.

22. Fr. Knopper, ouvr. cité, p. 507. 
s'indigne par exemple de la mode littéraire de ses contemporains qui diffusent les légendes exaltant la rudesse des chevaliers du Moyen Âge et attisant le sens de la révolte chez les jeunes gens; il met ses pairs en garde contre une telle tentation et prend part à un débat entre savants viennois sur la présence de templiers à Mödling, hypothèse qu'il réfute bien évidemment.

La sobriété des sentiments est de rigueur chez lui, bien qu'il laisse entrevoir des indices de contamination par l'esthétique du chantre des Alpes, Haller. Ce n'est donc nullement la nouvelle esthétique du premier romantisme allemand qui l'influence. Son éloge des habitants des Alpes ne sert qu'à souligner que les sources du contentement seraient "sobriété, travail et vertu" ${ }^{23}$ ». Son goût pour les vastes panoramas s'exprime par des épithètes laconiques : «superbe, romantique, beauté surnaturelle» ("herrlich, romantisch, überirdische Schönheit»). Leuphorie qui le gagne en haut d'un rocher qu'il escalade près de Vienne en se rendant aux thermes de Baden est instantanément récupérée à des fins didactiques : "Tout homme qui doute de la toute-puissance du Créateur, qui ignore les merveilles de ses ouvres, se doit de venir ici et contempler ce paysage ${ }^{24}$.» Croisant des pèlerins en Styrie, il reste maître de ses émotions :

Les chalets épars des montagnards, les silhouettes du [...] Höcherberg enneigé, les passants sur le chemin, l'écho des cantiques à Marie, sont des beautés que l'on peut ressentir mais pas décrire ${ }^{25}$.

Le point culminant de son récit est sa visite de la célèbre église de pèlerinage de Mariazell en Styrie mais, après un bref élan d'euphorie mystique, il est agacé par les bondieuseries. Il achète une brochure narrant les miracles, signale avec exaspération qu'elle a été publiée en 176I, à la grande époque de l'Aufklärung, la trouve «indigeste» et ne tarde pas à se plonger avec délice dans la lecture d'un ouvrage historique.

Ces genres d'excursions avaient donc la vocation de rappeler inlassablement que la connaissance des provinces était un acte patriotique. Arpenter les lieux permettait de maîtriser la topographie, peut-être à des fins militaires, et d'observer les mentalités, pour conseiller les réformateurs.

\section{Voyage et popularité des archiducs}

Les princes des petites cours allemandes avaient assidûment cultivé tout au long du XVIII ${ }^{\mathrm{e}}$ siècle la tradition du Grand Tour ${ }^{26}$. Dans le cas des archiducs

23. J. C. Unger, Reise durch österreichische und steyerische Gebirgsgegenden. Ein Beitrag zur österreichischen Länderkunde, Vienne, Pichler, I803, p. I39.

24. Ibid., p. 29.

25. Ibid., p. I44

26. J. Rees, W. Siebers, H. Tilgner (éd.), Europareisen politisch-sozialer Eliten im I8. Jahrhundert. 
d'Autriche, un déplacement était plus délicat puisqu'il présupposait un respect de l'étiquette. C'est pourquoi l'empereur Joseph II voyagea incognito sous le nom de comte de Falkenstein, et était irrité par l'accueil que les notables lui réservaient bien que ses visites ne fussent pas officielles. Cet empereur, mort en I790, a érigé la mobilité en devoir politique; outre ses voyages en France et en Italie, il a inspecté plusieurs fois ses provinces; les relations en étaient consignées dans ses lettres à Marie-Thérèse ou confiées à l'un de ses accompagnateurs. Les archiducs, au XIX ${ }^{\mathrm{e}}$ siècle, conserveront cette tradition du voyage. L'un de ses neveux, l'archiduc Jean, fut le premier à marcher sur ses traces et, comme on va le voir, il illustra avec talent les orientations et les pratiques de la nouvelle génération.

Né en 1782 à Florence, mort en 1859 à Graz, l'archiduc Jean a joui et jouit encore d'une popularitéér qui est à attribuer à plusieurs facteurs. Au côté romantique de son existence, puisqu'il épousa une roturière, Anna Plochl, fille d'un aubergiste de Bad Aussee. À son caractère frondeur et aux critiques qu'il osa formuler contre la politique menée par son frère l'empereur François Ir d'Autriche. Enfin, aux réformes importantes qu'il mit en place pour moderniser les territoires qui lui furent confiés, à savoir d'abord le Tyrol puis la Styrie, dont il stimula et accompagna l'industrialisation. Lui-même avait reçu une formation d'ingénieur des armées; il assura le commandement d'un régiment en I800 et son armée fut battue en I80I, à Hohenlinden, par Moreau. Après la paix de Lunéville, il fut nommé gouverneur du Tyrol, encouragea les Tyroliens et les habitants du Vorarlberg à prendre les armes contre Ney; il remporta la victoire du Strubpass le 3 novembre I8o5, mais ce succès fut remis en cause par Austerlitz, un mois plus tard. L'archiduc Jean consacra alors la majorité de son temps à arpenter la Styrie, qui était la plus grande province autrichienne, se faisant escorter de savants et d'artistes, soit d'environ une dizaine de personnes. Chargé en I809 du commandement de l'armée de l'Autriche intérieure, il répondit à l'appel de son frère l'archiduc Charles à une mobilisation générale, partageant son souhait de susciter un sursaut patriotique en Autriche. L'archiduc Jean avança jusqu'à Udine, remporta des victoires, puis dut se replier en Hongrie et arriva trop tard pour pouvoir participer à la bataille de Wagram. Il reprendra les armes en I8I5. À l'époque de la Restauration, il voyagera en Europe et se livrera à une forme d'espionnage industriel en Angleterre. Il érigera une ferme modèle, créera en

Theoretische Neuorientierung - kommunikative Praxis - Kultur - und Wissenstransfer, Berlin, Berliner Wissenschafts-Verlag, 2002.

27. Gr. Klingenstein (éd.), Erzherzog Johann von Österreich. Beiträge zur Geschichte seiner Zeit, Graz (Styrie), 1982; H. Magenschab, Erzherzog Johann. Habsburgs grüner Rebell, Graz (Styrie), 1982. 
I8I9 une des premières chambres d'agriculture, puis une École des mines, et plus tard un lycée technique à Graz, le Joanneum; il patronnera une association d'historiens et une de musiciens. Passant pour trop libéral, il sera tenu éloigné de Vienne par son frère l'empereur et Metternich. En définitive, l'originalité de ce personnage et de son parcours politique incitera les révolutionnaires de I848 à voir en lui un personnage providentiel et à lui confier la fonction honorifique, mais symbolique, de «Vicaire d'Empire».

Ses journaux de voyages, conservés aux archives de Vienne, étaient destinés seulement à ses proches, bien que de premiers extraits aient été publiés en I834, et la suite en I882. Les pages qui ont été écrites à l'époque qui nous intéresse décrivent une excursion faite dans les montagnes de Styrie en I8IO ${ }^{28}$. Elles constituent un exemple significatif de la réactivité de l'archiduc Jean à la mixité du genre et aux mutations qu'a connues l'écriture du voyage autour de I805-I8Io. Cette longue randonnée de I8Io, qui résulte d'une relative oisiveté forcée, relève aussi bien de la catégorie des excursions patriotiques que de celle des promenades délassantes.

L'auteur est doublement marginalisé : en tant que militaire, puisque son armée a été vaincue et la paix signée, et en tant qu'archiduc, car la cour de Vienne le trouve trop peu conformiste et lui-même estime qu'elle reste trop tournée vers le passé. Âgé de vingt-huit ans, il escalade sportivement les sommets des Alpes styriennes, selon la nouvelle mode, et visite les anciennes et illustres abbayes d'Admont ou Seckau, selon les habitudes des Aufklärer. Il herborise, collectionne les échantillons de roches, également dans la veine érudite. Il concentre son attention sur les paysans et les bergers, écoute leur musique et leurs chants, et s'intéresse à la situation économique dans un esprit réformateur. Il inspecte les mines de sel, s'informe sur les écoles et les églises. Tout cela caractérisait déjà les excursions patriotiques appréciées à l'époque des Lumières tardives, et l'archiduc s'inscrit ainsi dans la tradition de l'excursion de proximité entreprise à des fins instructives.

Mais il la transforme aussi à plusieurs égards parce que son texte est porté par une quête identitaire, tout à la fois personnelle et civique. Cet utilitarisme, hérité de l'Aufklärung, que nous avons relevé chez des fonctionnaires comme Schultes ou Unger, il est, lui, en mesure de le mettre en pratique à grande échelle : l'archiduc conçoit son exploration de la province comme, selon ses termes, une étape préparatoire au cadrage d'un futur travail "statistique», travail qu'il envisage de confier à un fonctionnaire, et qui verra effectivement le jour. Il repère quelles sortes de renseignements vaudront la peine d'être 
collectés et archivés : géographiques, économiques, ethnographiques, dialectaux. C'est pourquoi il visite tout, va partout, garde la visée encyclopédique des caméralistes du XVIII ${ }^{\mathrm{e}}$ siècle, et s'inspire de leurs rubriques. Certaines pages relèvent encore du genre dit des "voyages métallurgiques» et des descriptions de mines, qui avaient été commandités sous Marie-Thérèse.

Autre transposition : une des récentes ambitions des topographes et voyageurs autrichiens était de décrire les caractères provinciaux, afin de fournir des arguments favorables aux réformateurs, soucieux de promouvoir l'éducation, et aux intellectuels catholiques, exaspérés par les railleries des protestants. L'archiduc Jean prolonge cette ambition et il ajoute une critique de son cru, celle des usages en vogue à Schönbrunn. Même si la polémique est très retenue et le ton allusif, son amertume est décelable à plusieurs endroits : la psychologie de la population rurale, telle qu'il en fait l'éloge, est censée aider à restaurer les sentiments authentiques qu'il estime absents des relations citadines et mondaines. Les dernières lignes de son journal le confirment :

Mon voyage s'achève à Bruck, et avec lui mon journal; je rentre demain directement à Thernberg, tout à fait satisfait de ce que j'ai vu et observé, en excellente forme et le cœur gai, privilégié puisqu'il a fait constamment beau, avec des collections enrichies de nouveaux objets, une meilleure connaissance de ces régions, et enchanté d'avoir atteint l'objectif que je m'étais fixé pour ce voyage. Que de fois n’ai-je contemplé avec mélancolie le bonheur de maint paysan, les bienfaits que les habitants des Alpes tirent de leur ignorance des affaires du grand monde, le bonheur domestique, tout simplement, des montagnards. Las! pourquoi ce sort ne m’a-t-il pas été échu? - Cependant je vais supporter mon sort sans rechigner pour peu que réussissent les plans que je conçois pour le bien-être des gens et à condition que les talents que Dieu m'a octroyés, ainsi que mes forces physiques, ne restent pas inexploitées des années durant sans pouvoir être utiles à l'État ${ }^{29}$.

Transformer un journal de voyage en une chronique politisée et pamphlétaire n'était évidemment alors concevable que dans un journal intime non publié. La critique reste interne à la dynastie. Seul un initié percevra l'irritation de l'archiduc Jean quand il signale, et à deux reprises, que son frère l'empereur est attendu pour une partie de chasse à laquelle lui n'a pas été convié. La portée des remarques est plus générale s'il s'agit de constater les dysfonctionnements dans l'administration locale. L'archiduc ne précise pas si ce sont là ses observations personnelles ou s'il relaie les doléances de certains fonctionnaires, ce qui est d'autant plus probable que cela correspond au rôle de porte-parole qui incombait souvent aux voyageurs. Il est en tout cas le mieux placé pour pouvoir impunément déplorer que les petits fonctionnaires désobéissent aux ordres de leurs supérieurs, dysfonctionnements qu'il impute à la fainéantise des fonctionnaires viennois qui ne daignent pas sortir de la

29. Ibid., p. I4I. 
capitale et se comportent en «ronds-de-cuir sédentaires» («Stubensitzer»), lesquels seraient non seulement incompétents mais aussi indignes d'assumer leur responsabilité civique :

Puisqu'on ne peut pas empêcher qu'ils [les ouvriers de la mine de sel] volent du sel, il vaudrait mieux les laisser en emporter une quantité déterminée, la moralité du moins ne serait-elle plus en péril. Mais une telle mesure est inconcevable par ces messieurs les ronds-de-cuir sédentaires de la capitale ${ }^{30}$.

On voit se profiler l'homme d'État libéral que cet archiduc va devenir. Il désapprouve l'espionnite de son frère à l'affût de la moindre critique. Mais il n'idéalise pas les réformes joséphistes : il approuve la fermeture des couvents contemplatifs que son oncle avait ordonnée mais critique de ne pas les remplacer par d'autres institutions, ce qui aurait causé un retard dans l'instruction du peuple et tari les vocations dans le clergé autrichien. Sa position n'est donc plus seulement celle des joséphistes qui conseillaient de faire contrôler l'Église par l'État : grâce à sa prise en compte des réalités sociales populaires, l'archiduc Jean peut en effet ajouter les conclusions que lui inspirent les dégâts causés par la Révolution française et l'échec de l'Aufklärung. Et, en cela très proche de Pezzl qui avait pondéré son anticléricalisme en I803, l'archiduc note lors de son passage à Leoben :

La fermeture du séminaire général, qui fut une sage mesure de l'empereur Joseph, a eu des répercussions désastreuses. C'est ce qui se produit généralement quand on fait quelque chose de vraiment bon. Détruire est aisé, mais le remplacer par du meilleur est incomparablement plus difficile, si bien que, après avoir réalisé la première de ces opérations, l'état des choses s'aggrave parce qu'on n'a finalement plus rien du tout $t^{31}$.

L'aveu d'une frustration engendrée par un environnement dynastique dont il juge les méthodes archaïques, décalées, inefficaces, a-t-il été aggravé par une comparaison au "modèle» français sous Napoléon? Rien ne l'indique. Au contraire, l'archiduc a transposé sa frustration en ardeur patriotique et il ne laisse échapper aucune occasion pour le souligner. Citons par exemple sa rencontre d'un "maître de poste» en qui il semble avoir vu un alter ego du Tyrolien Andreas Hofer, ennemi patenté de Napoléon :

Je me rendis à pied à Kraubat et logeai au relais de poste; j'y trouvai un très brave homme en la personne du maitre de poste Eder; un vrai patriote, bon et franc; l'ennemi l'a beaucoup fait souffrir durant la dernière guerre, et il a accompli des actions au péril de sa vie, par exemple en libérant 300 prisonniers et en leur apportant de quoi survivre chez lui dans la montagne. J'ai entendu raconter des scènes effroyables concernant les pillages et les mœurs dépravées des Français. [...] Je me suis fait montrer, en chemin, le champ de bataille de Sankt Michael

30. Ibid., p. 9.

3I. Ibid., p. IO. 
et le monument qui y a été érigé; je mentionnerai le premier dans l'Histoire que j'écrirai de la guerre mais il vaudrait mieux détruire le second car il est absurde de construire un monument commémorant un malheur passé (p. 140).

Enfin et surtout, son journal reflète le changement qui avait affecté les relations de voyage qui devenaient polyphoniques, s'approchant, comme chez Arndt, du statut d'œuvre d'art totale. Le journal tenu par l'archiduc a un rythme primesautier; la succession fastidieuse des étapes est compensée grâce à des associations d'impressions ou d'idées, comme si la discontinuité de l'écriture avait libéré le chroniqueur et dynamisé la vivacité du raisonnement. Sans se sentir obligé de se justifier philosophiquement, le voyageur peut ainsi regrouper des considérations esthétiques, politiques et érudites.

L'archiduc esthétise ses critères en matière de sociabilité et de tourisme. Il reste rousseauiste, a des certitudes morales, recherche le parallélisme entre l'âme et la nature : son idéal est de favoriser la formation et l'épanouissement de l'individu, lequel mettra ensuite ses qualités au service de la communauté. C'est pourquoi, exactement comme tous les voyageurs l'avaient fait à l'époque de l'Aufklärung, il rapporte le déroulement de ses conversations avec les érudits locaux et cite leurs noms. Mais il ne s'en contente pas et privilégie en fait la description de scènes rurales : une modification majeure par rapport aux fonctionnaires dont il a été question plus haut est l'accent que ce journal met sur la diversité des coutumes paysannes. L'archiduc détaille la diversité des habits folkloriques, des jeux et des danses, de l'habitat, il dessine luimême. Mais il serait vain de chercher des critères philosophiques dans ce texte : ce qui est ainsi montré, ce sont des régionalismes qui, selon l'archiduc, seraient l'essence et la richesse du patrimoine autrichien, et une manifestation de patriotisme qui était encore une fois une provocation et un reproche en direction de Vienne.

Il esthétise aussi la pratique du tourisme. Il prise l'engagement sportif, l'implication physique, les traversées risquées des glaciers et des torrents, les sensations fortes, le goût du sublime et les porte à leur plus haut niveau. Toutes proportions gardées, les scènes décrites rappellent le célèbre tableau de Caspar David Friedrich, Der Wanderer. Les effusions de l'archiduc résultent d'une observation attentive du monde extérieur, d'un processus introspectif et d'une spiritualisation de l'argumentation. Sa formulation en est certes sommaire mais elle démontre que l'auteur réunissait les conditions nécessaires pour devenir le mécène d'artistes romantiques et en particulier du peintre viennois Karl Russ (1779-I843) qui l'accompagnait et dessinait ces paysages. 
Pour relater son séjour à Vienne à la veille de l'arrivée de Napoléon, on pouvait arriver d'Allemagne du Nord, marquer sa surprise face à une aire culturelle spécifique, comme la génération de Nicolai l'avait fait précédemment, mais en y associant dorénavant le sceau d'une personnalité haute en couleurs : Arndt impose un ton frondeur, Reichardt celui d'un expert. On trouve également des plaidoyers pro domo rédigés par des fonctionnaires studieux, professeurs, médecins, qui s'érigeaient en porte-parole d'une bourgeoisie viennoise aisée, disposée à se distraire mais sans s'encanailler, continuant à se réclamer du joséphisme et d'une Aufklärung catholique mais loyalement monarchiste et nullement révolutionnaire.

Si hétérogènes soient-ils, les quelques exemples de récits de voyages en Autriche évoqués ici attestent l'absence de rupture ou de confrontation entre les générations. La préservation de centres d'intérêt et de pratiques d'écriture, l'éloge du mécénat, des fêtes, de l'érudition, en ont fourni la preuve. Vienne restait un haut lieu de la légitimité dynastique où il faisait bon vivre. Des mutations s'esquissent cependant par le biais des émotions esthétiques face aux paysages et dans les passages satiriques qui expriment l'irritation face aux imperfections de la collectivité et la souffrance de personnalités sensibles confrontées à leur propre complexité. Ces voyageurs, quand ils combattaient ce qu'ils traitaient de sclérose institutionnelle ou de nonchalance viennoise, participaient - mais avec circonspection - à une effervescence d'idées et de sentiments patriotiques qui, ici, ne faisaient pas abstraction d'un arrière-plan militaire. 